\title{
O IMPACTO DO CURSO DE NIVELAMENTO NO DESEMPENHO EM CÁLCULO 1 DE RECÉM-INGRESSOS NO CURSO DE ENGENHARIA CIVIL DA UNIVERSIDADE FEDERAL DE ALAGOAS
}

Alícia Caroline de Lima Silva-alicia.lima@ctec.ufal.br Universidade Federal de Alagoas, Centro de Tecnologia Av. Lourival Melo Mota, S/N, Tabuleiro do Martins

57072-970 - Maceió - Alagoas

Diana Maria Pereira Caires - diana.caires@ctec.ufal.br Universidade Federal de Alagoas, Centro de Tecnologia Av. Lourival Melo Mota, S/N, Tabuleiro do Martins 57072-970 - Maceió - Alagoas

Mariana Karla Guedes da Silva Loz_kmarianaloz@gmail.com Universidade Federal de Alagoas, Centro de Tecnologia Av. Lourival Melo Mota, S/N, Tabuleiro do Martins 57072-970 - Maceió - Alagoas

Gabriel de Carvalho Freitas - xgabrielcfreitas@gmail.com Universidade Federal de Alagoas, Centro de Tecnologia Av. Lourival Melo Mota, S/N, Tabuleiro do Martins 57072-970 - Maceió - Alagoas

Roberto Barbosa dos Santos - rbs@ctec.ufal.br Universidade Federal de Alagoas, Centro de Tecnologia Av. Lourival Melo Mota, S/N, Tabuleiro do Martins 57072-970 - Maceió - Alagoas

Resumo: Ao passar dos anos, têm sido observados elevados índices de evasão e retenção de estudantes no curso de Engenharia Civil do Campus A. C. Simões da Universidade Federal de Alagoas. Nesse contexto, uma das maiores taxas de reprovação de discentes é referente à disciplina de Cálculo 1, apresentada aos alunos no primeiro período do curso e considerada uma das mais complexas do ciclo básico. Diante disso, o Curso de Nivelamento das Engenharias foi concebido e é realizado anualmente desde 1990 com o intuito de auxiliar a adaptação dos recém-ingressos ao ambiente universitário, visando colaborar na redução dos índices mencionados. Posto isso, o propósito deste estudo é investigar a repercussão dessa proposta, por meio da análise dos dados de desempenho de turmas de estudantes recémingressos na disciplina de Cálculo 1 do curso em questão, referentes às turmas dos anos de 2011 a 2018. Foi empregado um teste de hipótese a fim de investigar a relação entre a participação no Curso de Nivelamento e a nota final obtida na disciplina de Cálculo 1. Os resultados das análises performadas sugerem uma tendência de os participantes da atividade apresentarem desempenho superior aos não participantes em relação a parâmetros como índices de evasão e retenção e nota final na disciplina.

Palavras-chave: Curso de Nivelamento. Engenharia Civil. Cálculo. Teste de hipótese. 


\section{INTRODUÇÃO}

\subsection{Histórico da disciplina de Cálculo 1}

$\mathrm{Na}$ Universidade Federal de Alagoas (UFAL), a disciplina de Cálculo 1, mais especificamente no curso de graduação em Engenharia Civil do Campus A. C. Simões - foco dessa análise - sempre foi muito estigmatizada. Essa percepção decorre, principalmente, dos seus elevados índices de reprovação, que vem sendo observados com o passar dos anos.

Alguns dos fatores que podem desencadear essa situação têm início já no processo de entrada desses estudantes na universidade: no caso da UFAL, o curso de Engenharia Civil possui ingresso estudantil através da nota no Exame Nacional do Ensino Médio (ENEM). Entretanto, percebe-se que o nível dos conhecimentos, ao menos no que diz respeito às áreas de exatas, que é abordado no ensino médio e, consequentemente, cobrado nesse exame não é compatível com o requerido na disciplina de Cálculo 1. Dessa forma, alguns dos recémingressos no curso não possuem a base de conhecimentos necessária e presumida para cursar essa matéria. Outro aspecto a ser levado em consideração é o fato dessa disciplina ser apresentada no primeiro período do curso, no qual o estudante está em fase de adaptação ao ambiente universitário.

Nesse cenário, é comum observar altos índices de desistência na disciplina. Segundo estudos realizados por Rodrigues Neto et al. (2018) acerca da realidade do curso, a retenção dos estudantes é prejudicial tanto por atrasar a conclusão do curso quanto por desmotivar diversos estudantes, os quais, dependendo de suas realidades, acabam por trancar a matrícula ou desistir do curso.

Assim, o que é notado há muito tempo é que a disciplina de Cálculo 1 é determinante para a continuidade dos discentes até o fim do curso. Segundo estudos realizados por docentes da instituição sobre a mesma base de dados utilizada neste trabalho, as chances de um estudante de Engenharia Civil do campus A. C. Simões concluir o curso é maior se ele obtiver aprovação na matéria na primeira ou segunda tentativa, quando comparado aos que reprovam mais vezes nessa disciplina.

Os anos analisados no presente trabalho correspondem àqueles nos quais essa disciplina teve uma abordagem unificada, ou seja, o mesmo cronograma foi seguido pelos professores das diferentes turmas e as avaliações foram as mesmas para os discentes dos diferentes cursos envolvidos. Essa conduta busca nivelar a formação dos discentes na matéria em questão, com o intuito de eliminar possíveis variações entre as turmas, diferindo apenas em relação aos professores que a ministram.

\subsection{Funcionamento do Curso de Nivelamento das Engenharias}

O Nivelamento das Engenharias teve início em 1990, sendo uma atividade proposta e criada pelo Programa de Educação Tutorial (PET) de Engenharia Civil da UFAL, a qual tem sido realizada até hoje com o apoio de vários outros grupos discentes do Centro de Tecnologia (CTEC). Apesar de já ter ocorrido semestralmente, na grande maioria das vezes a atividade ocorre anualmente, com ambas as turmas ingressantes (com ingresso de periodicidade semestral) como público-alvo. Ademais, geralmente consiste em duas semanas de atividades com finalidade de amenizar e permitir uma transição mais suave entre ensino médio e superior para os participantes. Nesse contexto, além de aulas de matemática abordando os principais conteúdos básicos que serão exigidos ao longo do curso, ministradas por estudantes veteranos e/ou professores convidados, são realizadas dinâmicas, palestras, visitas técnicas, exposições 
laboratoriais e atividades lúdicas com o intuito de apresentar o ambiente universitário e promover a integração dos estudantes.

Além disso, um dos principais diferenciais de uma universidade são as inúmeras possibilidades que são oferecidas, ainda durante o curso. Nesse sentido, o Nivelamento para o curso de Engenharia Civil tem como um de seus principais objetivos apresentar os diversos grupos discentes dos quais o estudante recém-ingresso pode participar, como o Centro Acadêmico de Engenharia Civil (CAEC), a Empresa Júnior de Engenharia Civil e Arquitetura (EJEC), os grupos PET, a Associação Atlética Acadêmica de Engenharia Civil (AAAEC), o Programa Especial de Capacitação Discente (PEC), entre outros, bem como oportunidades de projetos de Iniciação Científica, monitorias e afins.

Segundo Almeida e Godoy (2016), existe uma estreita relação entre evasão e reprovação nas disciplinas do ciclo básico, em especial a disciplina de Cálculo Diferencial e Integral: cerca de $34 \%$ dos motivos de evasão devem-se às reprovações sucessivas em matérias deste ciclo. Tal fato deixa uma quantidade expressiva de vagas ociosas nas instituições. Portanto, momentos de cunho motivacional, como palestras e depoimentos de estudantes em final de curso, bem como de graduados, são preparados no curso de Nivelamento com a finalidade de estimular os estudantes a se interessarem pelo curso e contribuir para o decréscimo dos índices citados.

\subsection{Objetivo geral}

Tomando como foco os estudantes recém-ingressos no Curso de Engenharia Civil do campus A. C Simões da UFAL, o intuito deste trabalho é analisar o impacto relacionado à participação desses estudantes no Curso de Nivelamento das Engenharias no seu desempenho na disciplina "Cálculo 1".

Os estudos aqui desenvolvidos têm por finalidade contribuir para a melhoria das ações propostas no Nivelamento das Engenharias, propiciando, assim, uma transição mais natural entre ensino médio e o superior para os estudantes ingressantes, culminando na melhoria da qualidade do curso de graduação abordado.

\section{METODOLOGIA}

A metodologia empregada no estudo aqui exposto consistiu numa análise estatística a partir de dados de desempenho de diversas turmas na disciplina de "Cálculo 1". Os dados e informações adquiridas foram disponibilizados pelo Núcleo de Tecnologia da Informação (NTI) da Universidade Federal de Alagoas (UFAL), órgão responsável por prover o suporte na área de tecnologia da informação na instituição. Em um primeiro momento, os dados foram disponibilizados a pedido do então vice-diretor da unidade acadêmica, Prof. Roberto Barbosa dos Santos e, em seguida, concedidos para a análise deste estudo.

A partir dessas informações, alguns filtros foram aplicados à extensa gama de dados com o intuito de realizar uma análise restrita ao objetivo deste trabalho. Inicialmente, a amostra foi restringida às informações de 12 turmas distintas, a partir do primeiro semestre do ano letivo de 2011 até o segundo semestre do ano letivo de 2018. Posteriormente, para conferir maior precisão às análises e conclusões feitas, foram analisados os dados apenas das turmas cujas aulas foram ministradas por um único docente selecionado, por ter ministrado aulas ao maior número de turmas dentro do histórico de docentes da disciplina.

O critério utilizado para considerar que o estudante compareceu ao Nivelamento foi a assiduidade mínima de $75 \%$ nas atividades, justificando a validade da experiência do aluno na atividade, da forma como foi proposta. 
Por fim, foi realizado um teste de hipótese para comparar as notas finais médias dos participantes e não participantes da atividade, a fim de verificar se há correlação entre a participação no Nivelamento e notas finais superiores na disciplina. A ferramenta aplicada foi o Teste T de Student, cuja estatística de teste padronizada está exposta na Equação (1), que considera as médias amostrais $\left(\overline{x_{1}}, \overline{x_{2}}\right)$ e populacionais $\left(\mu_{1}, \mu_{2}\right)$, além do erro padrão $\left(s_{\overline{x_{1}}}-\overline{x_{2}}\right)$.

$$
t=\frac{\left(\overline{x_{1}}-\overline{x_{2}}\right)-\left(\mu_{1}-\mu_{2}\right)}{s_{\overline{x_{1}}-\overline{x_{2}}}}
$$

Devido ao tamanho das amostras, o teste foi realizado utilizando o recurso de análise de dados "Teste T: duas amostras presumindo variâncias equivalentes" do software Microsoft Excel.

\section{RESULTADOS}

De posse dos dados de desempenho dos discentes, bem como da participação no Nivelamento, foram feitas diversas análises. Inicialmente foi quantificada a participação dos discentes de cada turma no Nivelamento, conforme exposto na Tabela 1.

Tabela 1 - Presença dos discentes de cada turma no

Nivelamento.

\begin{tabular}{c|c|c|c|}
\hline $\begin{array}{c}\text { Período letivo da } \\
\text { turma }\end{array}$ & $\begin{array}{c}\text { Total de discentes } \\
\text { ingressantes }\end{array}$ & $\begin{array}{c}\text { Número de discentes } \\
\text { presentes no } \\
\text { Nivelamento }\end{array}$ & $\begin{array}{c}\text { Percentual de discentes } \\
\text { presentes no } \\
\text { Nivelamento }\end{array}$ \\
\hline 2011.1 & 40 & 30 & $75,00 \%$ \\
\hline 2011.2 & 34 & 17 & $50,00 \%$ \\
\hline 2012.1 & 35 & 20 & $57,14 \%$ \\
\hline 2012.2 & 39 & 4 & $10,26 \%$ \\
\hline 2013.1 & 35 & 19 & $54,29 \%$ \\
\hline 2013.2 & 35 & 22 & $62,86 \%$ \\
\hline 2016.1 & 35 & 20 & $57,14 \%$ \\
\hline 2016.2 & 28 & 8 & $28,57 \%$ \\
\hline 2017.1 & 36 & 20 & $55,56 \%$ \\
\hline 2017.2 & 30 & 10 & $33,33 \%$ \\
\hline 2018.1 & 38 & 22 & $57,59 \%$ \\
\hline 2018.2 & 32 & 16 & $50,00 \%$ \\
\hline
\end{tabular}

Fonte: Autores (2020)

Analisando os dados apresentados na Tabela 1 nota-se que a adesão ao Nivelamento por parte dos discentes que ingressam no primeiro semestre do ano letivo (com terminação ".1") é, na maioria dos casos estudados, significativamente maior que as turmas de ingresso posterior, com exceção do ano de 2013, em que o cenário foi inverso porém com uma diferença pequena entre as turmas, com participação da turma 2013.2 8,57\% superior à 2013.1.

É importante ressaltar que desde o ano de 2011 a UFAL adota como meio de ingresso à Instituição o Sistema de Seleção Unificada (SiSU). Entretanto, todas as vagas são ofertadas no mesmo período, ou seja, a composição das duas turmas é feita no início do ano, visto que a Universidade não adere ao SiSU do segundo semestre. Posto isso, o número inferior de 
(C) COBENCE 2020

"Os desafios para formar hoje o engenheiro do amanhã"

participantes do Nivelamento integrantes de turmas ".2" em relação às "..1" pode ser causado por um baixo interesse na atividade devido à falta de motivação, visto que, para esses alunos, o início das aulas no curso superior só ocorre cerca de 6 meses após a realização do Nivelamento.

De modo a compreender de maneira objetiva um dos impactos diretos do curso de Nivelamento, foram comparadas, para cada uma das turmas estudadas, o índice de aprovação geral na disciplina de Cálculo 1 e a taxa de aprovação dos discentes presentes na atividade oferecida em seus respectivos anos de ingresso. Essa comparação está exposta na Figura 1.

Figura 1 - Gráfico comparativo da taxa de aprovação de cada turma com a taxa de aprovação dos discentes participantes do Nivelamento na disciplina de Cálculo 1.

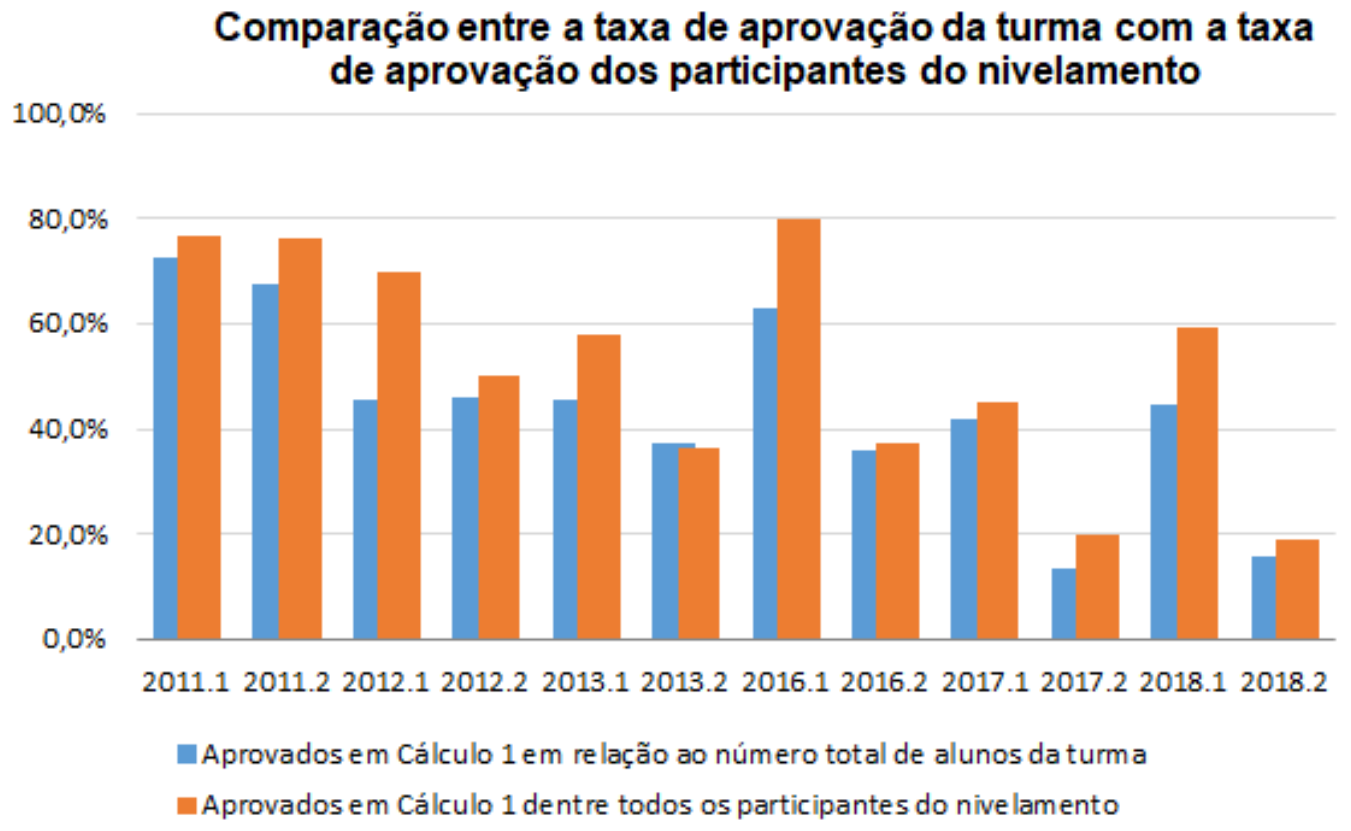

Fonte: Autores (2020).

Segundo a Figura 1, a taxa de aprovação dos participantes do nivelamento é superior à taxa de aprovação geral da turma em 11 das 12 turmas analisadas, destacando-se o semestre letivo 2012.1, no qual o desempenho médio dos participantes do Nivelamento foi $24,29 \%$ superior ao desempenho médio da turma em geral. Tem-se uma exceção em 2013.2, na qual a diferença foi de apenas $0,78 \%$. Dessa forma, sugere-se uma correlação entre a participação no Nivelamento e uma melhor performance em Cálculo 1, podendo ser justificada pelas aulas de matemática básica ministradas ao longo da atividade, que servem de base para os assuntos desenvolvidos na disciplina.

Calculando-se a média dos dados expostos na Figura 1, obtém-se uma comparação direta entre as taxas médias de aprovação nos dois cenários, considerando as turmas em geral e considerando apenas os discentes presentes no curso de Nivelamento, conforme exposto na Figura 2. 
CCOBENCE 2020 (C)

"Os desafios para formar hoje o engenheiro do amanhã"
$\mathrm{O1}$ a $\mathrm{O3}$ de dezembro Evento On-line

Figura 2 - Gráfico comparativo entre a taxa média de aprovação dos participantes do Nivelamento e de aprovação média das turmas.

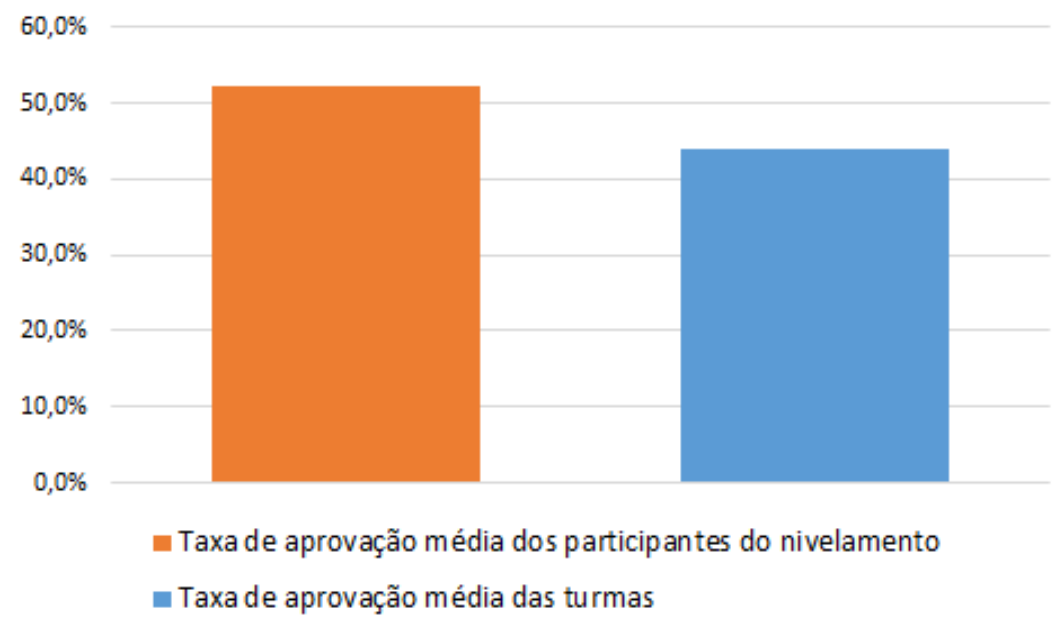

Fonte: Autores (2020).

Assim, para as turmas analisadas, a taxa de aprovação média em Cálculo 1 dos participantes do Nivelamento é $8,24 \%$ superior à das turmas em geral.

Filtrando os dados para obter um enfoque diferenciado, foi estudado também o desempenho dos participantes do Nivelamento dentro dos grupos de aprovados e reprovados na disciplina. Essa verificação está indicada na Figura 3.

Figura 3 - Gráfico do desempenho dos participantes do Nivelamento, em relação ao desempenho geral de cada turma.

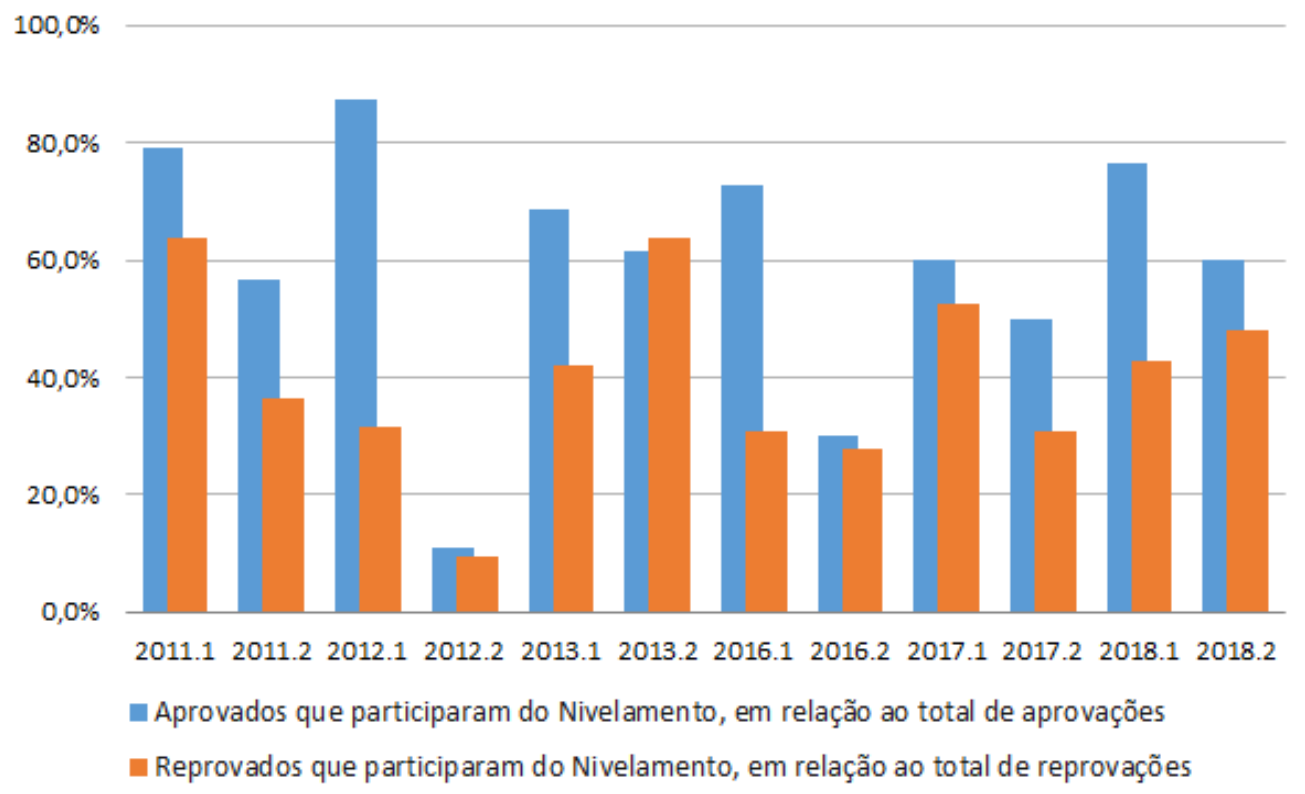

Fonte: Autores (2020). 
Observa-se que, na maioria das turmas, a porcentagem de participantes do Nivelamento aprovados, em relação ao total de aprovações, é maior que a de reprovados. Novamente, a única exceção é a turma 2013.2, na qual a porcentagem de reprovados supera a de aprovados por $2,10 \%$.

Ademais, em se tratando da comparação entre aprovados e reprovados na disciplina que estiveram presentes na atividade, em relação ao desempenho geral, a diferença é bastante expressiva em algumas turmas, podendo ser citadas 2012.1, 2016.1 e 2018.1, em que a porcentagem de aprovados supera a de reprovados em 55,92\%, 41,96\% e 33,61\%, respectivamente. Percebe-se, portanto, que, na maioria das turmas, os participantes do Nivelamento representam uma grande parcela do número de aprovados, enquanto que o inverso ocorre para o número de reprovações.

Considerando o alto índice de reprovações por falta na disciplina de Cálculo 1 e que essas reprovações tenham ocorrido em consequência da grande dificuldade enfrentada pelos alunos no primeiro período letivo, o que leva à desistência antes mesmo de ocorrerem as últimas provas, analisou-se também como a participação no curso de Nivelamento contribuiu para a conclusão da disciplina (ou seja, a não reprovação por falta) em cada turma, cujos resultados podem ser visualizados na Figura 4.

Figura 4 - Gráfico comparativo da taxa de concluintes da disciplina de cada turma com a taxa de concluintes da disciplina dos discentes participantes do Nivelamento.

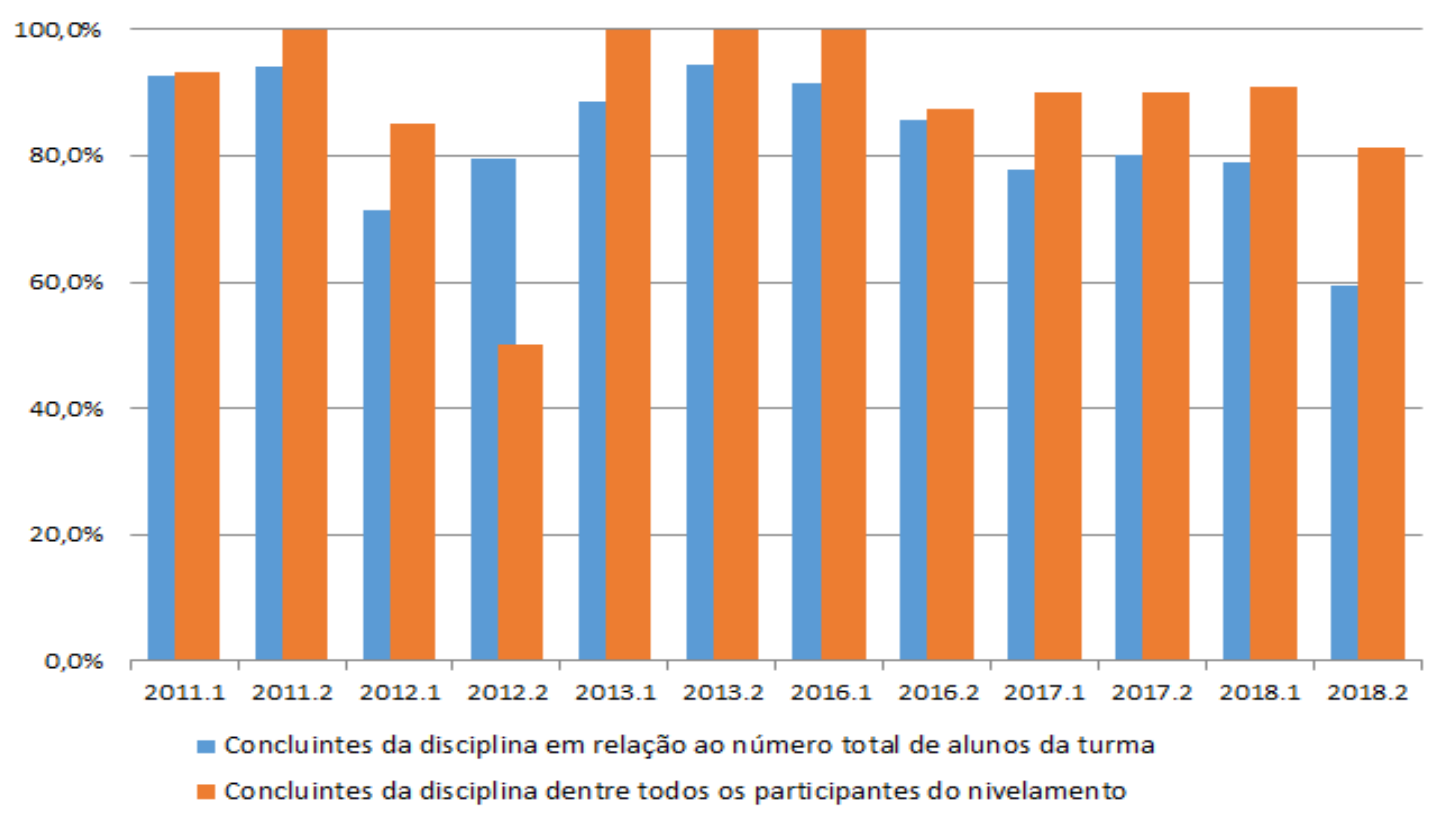

Fonte: Autores (2020).

Dessa forma, foi possível constatar que, em média, somente 19,70\% dos desistentes por falta são participantes do Nivelamento. Além disso, é possível observar na Figura 4 que em todas as turmas, exceto na de 2012.2, a taxa de concluintes é maior entre os discentes que participaram do Nivelamento. Ademais, em 4 das 12 turmas, nenhum participante da atividade reprovou por falta na disciplina. Dessa maneira, sugere-se que o cunho motivacional da atividade pode ter contribuído para a obtenção dos resultados apresentados. 
Para comparar quantitativamente a diferença de desempenho entre participantes e não participantes da atividade, as notas finais de cada discente integrante desses dois grupos foram categorizadas em duas amostras e, posteriormente, calculou-se a média aritmética de cada uma delas, conforme mostrado na Tabela 2.

Tabela 2 - Médias calculadas a partir das notas finais dos discentes participantes e não participantes do Nivelamento.

\begin{tabular}{c|c|c|c|}
\hline Amostra & Descrição & $\begin{array}{c}\text { Número de notas } \\
\text { finais (n) }\end{array}$ & Média da amostra \\
\hline 1 & $\begin{array}{c}\text { Notas finais de } \\
\text { participantes }\end{array}$ & 208 & 5,11 \\
\hline 2 & $\begin{array}{c}\text { Notas finais de não } \\
\text { participantes }\end{array}$ & 209 & 3,34 \\
\hline
\end{tabular}

Fonte: Autores (2020).

É possível constatar que a média da amostra 1 é superior à segunda, apresentando uma diferença percentual de 52,91\%. Entretanto, optou-se por realizar um teste de hipótese, de acordo com Larson e Farber (2015), para subsidiar estatisticamente a afirmação de que discentes que participam do curso de Nivelamento têm, em média, notas finais maiores do que os não participantes na disciplina de Cálculo 1 .

Considerando que se tratam de duas amostras independentes, ambas com $n>30$, com desvio populacional desconhecido, para realizar o teste de hipótese foi utilizado o Teste $\mathrm{T}$ de Student para a diferença entre duas médias, com nível de significância de 5\%, amplamente utilizado na literatura.

A princípio, foi utilizado o Teste $\mathrm{F}$ para inferência entre duas variâncias e foi constatado com $95 \%$ de nível de confiança que, neste caso, as variâncias das duas amostras são equivalentes.

Como hipótese nula para o Teste T supôs-se que as médias são iguais, logo, em caso de rejeição dessa hipótese, a alternativa é que elas são diferentes. Os dados do teste, obtidos a partir do software Microsoft Excel, estão dispostos na Tabela 3.

Tabela 3 - Dados considerados e calculados por meio do Teste.

\begin{tabular}{c|c|c|c|}
\hline $\begin{array}{c}\text { Nível de } \\
\text { significância }\end{array}$ & $\begin{array}{c}\text { Graus de liberdade } \\
\text { (g.l.) }\end{array}$ & T crítico & $\begin{array}{c}\text { Estatística de teste } \\
\text { calculada }\end{array}$ \\
\hline $5 \%$ & 415 & 1,9656 & 6,271 \\
\hline \multicolumn{2}{r}{}
\end{tabular}

Sendo o teste bilateral com g.l. $=415$, as regiões de rejeição são $t<-1,9656$ e $t>1,9656$. Como mostra a Tabela 3, a estatística de teste padronizada calculada resultou em $\mathrm{t}=6,271$. Sendo assim, por estar na área de rejeição, rejeitou-se a hipótese nula.

Consequentemente, nota-se evidência suficiente, ao nível de significância de 5\%, para rejeitar a hipótese nula de que as médias das amostras são iguais. Por conseguinte, sugere-se que a média do desempenho dos participantes dos discentes que participam do Nivelamento é maior que a dos não participantes.

\section{CONSIDERAÇÕES FINAIS}

A partir das análises dos dados coletados, observou-se que a taxa de aprovação e as notas finais dos estudantes na disciplina de Cálculo 1 são, em geral, maiores entre os discentes que 
estiveram presentes no nivelamento do que entre os não participantes, sugerindo um impacto positivo das aulas, provas e demais atividades realizadas no Curso de Nivelamento no desempenho dos estudantes na disciplina.

Vale ressaltar ainda que os resultados de taxas de evasão e retenção menores entre os participantes da atividade evidenciam uma possível correlação entre a participação no Curso de Nivelamento e maior motivação por parte dos estudantes, o que sugere eficácia nas ações de cunho motivacional (palestras, dinâmicas, visitas técnicas e laboratoriais, conversas com egressos e estudantes em final de curso etc.) propostas na atividade.

Além disso, a apresentação do curso de Engenharia Civil e dos demais grupos discentes presentes na unidade acadêmica (CAEC, EJEC, grupos PET, AAAEC, PEC, entre outros), bem como das oportunidades de projetos de Iniciação Científica e monitorias, contribui para uma maior participação dos estudantes recém-ingressos nesses grupos, motivando-os a permanecer no curso e os proporcionando uma formação mais completa, ao passo que fortalece esses programas e reafirma o caráter formador das universidades ao fomentar suas esferas de atuação, como o ensino, a pesquisa e a extensão.

Assim, com base nas análises performadas, sugere-se que o Curso de Nivelamento das Engenharias organizado pelo PET Engenharia Civil da UFAL em conjunto com os demais grupos discentes do CTEC tem impactado positivamente o desempenho dos estudantes que ingressam no Curso de graduação em Engenharia Civil da UFAL na disciplina de Cálculo 1, o que geralmente surte efeitos positivos ao longo de toda a graduação.

\section{Agradecimentos}

Ao Fundo Nacional de Desenvolvimento da Educação (FNDE) do Ministério da Educação (MEC) pelo financiamento do PET, assim como aos demais grupos discentes que tornam possível a realização anual do Nivelamento das Engenharias.

\section{REFERÊNCIAS}

ALMEIDA, E. de; GODOY, V. E. A Evasão nos Cursos de Engenharia: uma análise a partir do COBENGE. In: XLIV Congresso Brasileiro de Educação em Engenharia, 2016, Rio Grande do Norte. Anais. Natal, 2016.

LARSON, Ron; FARBER, Betsy. Estatística Aplicada. São Paulo: Pearson Education do Brasil. 2015.

RODRIGUES NETO, E. et al. O Impacto que o Curso de Nivelamento traz aos RecémIngressos no Curso de Engenharia Civil da Universidade Federal de Alagoas. In: XLVI Congresso Brasileiro de Educação em Engenharia e $1^{\circ}$ Simpósio Internacional de Educação em Engenharia da ABENGE, 2018, Bahia. Anais. Salvador, 2018. 


\title{
IMPACT OF THE LEVELING COURSE ON THE PERFORMANCE ON CALCULUS 1 OF NEWLY ADMITTED CIVIL ENGINEERING STUDENTS OF THE FEDERAL UNIVERSITY OF ALAGOAS
}

\begin{abstract}
Over the years, high dropout and student retention rates in the Civil Engineering course at the A. C. Simões campus of the Federal University of Alagoas have been observed. In this regard, one of the highest student failure rates is in the subject of Calculus 1, presented to pupils in the first semester and considered one of the most complex disciplines of the basic cycle. Therefore, the Engineering Leveling Course was conceived and has been carried out annually since 1990 with the aim of helping the adaptation of new students to the university environment, aiming to collaborate on the reduction of the mentioned indexes. Hence, the purpose of this study is to investigate the repercussion of this proposal through the analysis of the performance data of newly enrolled students on the Calculus 1 discipline of the mentioned course, referring to classes from the years 2011 to 2018. Hypothesis testing was used to investigate the relationship between participation in the Leveling Course and the final grade obtained in the Calculus 1 discipline. The results of the performed analysis suggest a tendency for the Leveling Course participants to perform better than non-participants in regards to parameters such as dropout and retention rates and final grade in the discipline.
\end{abstract}

Keywords: Leveling Course. Civil Engineering. Calculus. Hypothesis testing. 